\title{
Lateralizing the affected side of hippocampal sclerosis with quantitative high angular resolution diffusion scalars: a preliminary approach validated by diffusion spectrum imaging
}

\author{
Yi-He Wang ${ }^{1 \#}$, Zhen-Ming Wang ${ }^{2 \#}$, Peng-Hu Wei ${ }^{1}$, Chao Lu ${ }^{1}$, Xiao-Tong Fan ${ }^{1}$, Lian-Kun Ren ${ }^{3}$, \\ Yong-Zhi Shan ${ }^{1}$, Jie Lu ${ }^{2,4}$, Guo-Guang Zhao ${ }^{1,5}$ ^
}

${ }^{1}$ Department of Neurosurgery, Xuanwu Hospital, Capital Medical University, Beijing, China; ${ }^{2}$ Department of Radiology, Xuanwu Hospital, Capital Medical University, Beijing, China; ${ }^{3}$ Department of Neurology, Xuanwu Hospital, Capital Medical University, Beijing, China; ${ }^{4}$ Department of Nuclear Medicine, Xuanwu Hospital, Capital Medical University, Beijing, China; ${ }^{5}$ Center of Epilepsy, Beijing Institute for Brain Disorder, Beijing, China

Contributions: (I) Conception and design: J Lu, GG Zhao; (II) Administrative support: GG Zhao, LK Ren, YZ Shan; (III) Provision of study materials or patients: YH Wang, ZM Wang; (IV) Collection and assembly of data: YH Wang, PH Wei, C Lu, XT Fan; (V) Data analysis and interpretation: YH Wang, ZM Wang, PH Wei; (VI) Manuscript writing: All authors; (VII) Final approval of manuscript: All authors.

\#These authors contributed equally to this work.

Correspondence to: Jie Lu; Guo-Guang Zhao. Department of Neurosurgery, Xuan Wu Hospital, Capital Medical University, No. 45, Changchun Street, Xuanwu District, Beijing 100053, China. Email: imaginglu@hotmail.com; ggzhao@vip.sina.com.

\begin{abstract}
Background: Conflicts in regarding the lateralization of the seizure onset for mesial temporal lobe epilepsy (MTLE) are frequently encountered during presurgical evaluation. As a more elaborate, quantified protocol, indices of diffusion spectrum imaging (DSI) might be sensitive to evaluate the seizure involvement. However, the accuracy was less revealed. Herein, we determined the lateralizing value of the DSI indices among MTLE patients. Methods: Eleven MTLE patients were enrolled together with 11 matched health contrasts. All the participants underwent a DSI scan and with reconstruction of the diffusion scalar, including quantitative anisotropy (QA), isotropic (ISO), and track density imaging (TDI) values. Statistics of these indices were applied to identify the differences between the healthy and ipsilateral sides, and those between the patients and the controls, with special attention to areas of the crura of fornix (FORX), the parahippocampal radiation of the cingulum (PHCR), the hippocampus (HP), parahippocampus (PHC), amygdala (AM) and entorhinal cortex (EC).

Results: Regarding lateralization, TDI of the FORX and the PHCR reached an AUC value of 0.95 and 0.93, respectively $(\mathrm{P}<0.05)$, and QA, ISO, TDI of the PHCR, as well as TDI of the FORX were statistically significant amongst the laterals of the patients $(\mathrm{P}<0.05)$. Also, the QA of the PHCR were statistically different in the patients' ipsilateral side relative to the contrasts $(\mathrm{P}<0.017)$. The diffusion level on different grey matter structures were significantly decreased including HP, AM and EC in GQI space $(\mathrm{P}<0.017)$.

Conclusions: The quantitative diffusion scalars of the DSI, especially TDI of the FORX and the PHCR, are sensitive indices to define the ipsilateral side for MTLE patients. For preliminary exploration, the use of quantitative DSI scalars may help to improve the seizure outcome by increasing the accuracy of localization and lateralization for MTLE.
\end{abstract}

Keywords: Diffusion spectrum imaging (DSI); mesial temporal lobe epilepsy (MTLE); high angular quantitative analysis

Submitted Aug 06, 2020. Accepted for publication Nov 30, 2020.

doi: 10.21037/atm-20-5719

View this article at: http://dx.doi.org/10.21037/atm-20-5719

\footnotetext{
$\wedge$ ORCID: 0000-0002-7569-5252.
} 


\section{Introduction}

Mesial temporal lobe epilepsy (MTLE) is one of the most common types of human epilepsy. Presurgical evaluation is of immense importance in patients with MTLE because surgical treatment can provide seizure relief in $60-80 \%$ of the patients. However, $30-40 \%$ of patients with MTLE show negative magnetic resonance imaging (MRI) results, and the lateralization of the seizure in $1-15 \%$ cannot be determined only with scalp electroencephalogram (EEG) and MRI. It is necessary to optimize the noninvasive presurgical evaluation workflow for MTLE patients to provide less invasive surgery and better seizure outcome.

Comprehensive imaging modalities including functional imaging and structural imaging can provide lateralization or localization information for MTLE. Among them, diffusion MRI (dMRI) has played an important role in the presurgical evaluation of MTLE; it helps to determine the microstructural damage caused by neuronal loss at the seizure onset zone and allows the identification of the extra-focal regions connected to the seizure focus to be determined (1). Diffusion tensor imaging (DTI) has been widely used in the presurgical evaluation of patents with unilateral MTLE to assess the diffusion asymmetry between the two hemispheres (1-6). Nazem-Zadeh et al. have developed an important DTI-based response-driven lateralization model for MTLE, which helps to reduce diagnostic errors when determining laterality (7). However, DTI as one of the model-based methods cannot help determine the crossing and termination of fibers, and also generates multiple artifacts and false tracts, which may decrease its accuracy in the clinical settings (8).

Diffusion spectrum imaging (DSI) has been developed as a new, model-free method based on high angular resolution imaging, which can overcome the limitations of DTI $(9,10)$. Preliminary experiments with this procedure have showed the similarity of neuroanatomical features between primates and human $(10,11)$. Compared with DTI, DSI improves quantitative resolution with intra-voxel diffusion heterogeneity and does not resolve the average direction difference; moreover, to some extent, DSI quantifies the diffusion level more precisely than other methods. There are limited studies using DSI in the presurgical evaluation of the patients with MTLE. To the best of our knowledge, there were no reports using this high-resolution technique to investigate the heterogeneity of gray and white matter between the two sides of unilateral MTLE. In this study, we intend to (I) use DSI to quantitatively compare the microstructural differences of gray and white matter of the two sides in MTLE and (II) assess the reliability and effectiveness of DSI in lateralizing MTLE during presurgical evaluation. We present the following article in accordance with the STROBE reporting checklist (available at http://dx.doi.org/10.21037/atm-20-5719).

\section{Methods}

\section{Patients and controls}

A series of MTLE patients were recruited following the specific inclusion and exclusion criteria. The inclusion criteria were as follows: (I) unilateral MTLE, (II) DSI acquired with the same protocol, (III) diagnosis of MTLE by a multidisciplinary team (MDT) or postoperative pathology. The exclusion criteria were bilateral MTLE or other types of temporal lobe epilepsy and lack of DSI acquisition.

All the included patients have received the same presurgical evaluation. First, DSI and three-dimensional (3D) MRI imaging were performed after long-term video scalp EEG examination and detailed semiology investigation. Then, unilateral MTLE was defined by an MDT for both lateralization and localization. Finally, anterior temporal lobectomy (ATL) or stereoelectroencephalography (SEEG)guided radiofrequency thermocoagulation performed in these patients with unilateral MTLE. The final diagnosis was determined by MDT discussion or by SEEG results or pathological results.

For the control group, the inclusion criteria were as follows: (I) none of the controls had neurological or psychotropic disorders, (II) all the controls underwent the DSI scan following the same protocol, (III) age and sex be matched with the patient group. The exclusion criteria were healthy controls who did not agree to recruit or those with neurological or psychotropic disorders. A brief description of the characteristics of both patients and controls is presented in Table 1.

The study was conducted in accordance with the Declaration of Helsinki (as revised in 2013). The study was approved by the ethics committee at Xuanwu Hospital (NO. LYS [2019] 097) and informed consent was taken from all the patients.

\section{MRI and DSI acquisition}

All the raw data related to this study were acquired 
Table 1 Clinical data of the patients and controls

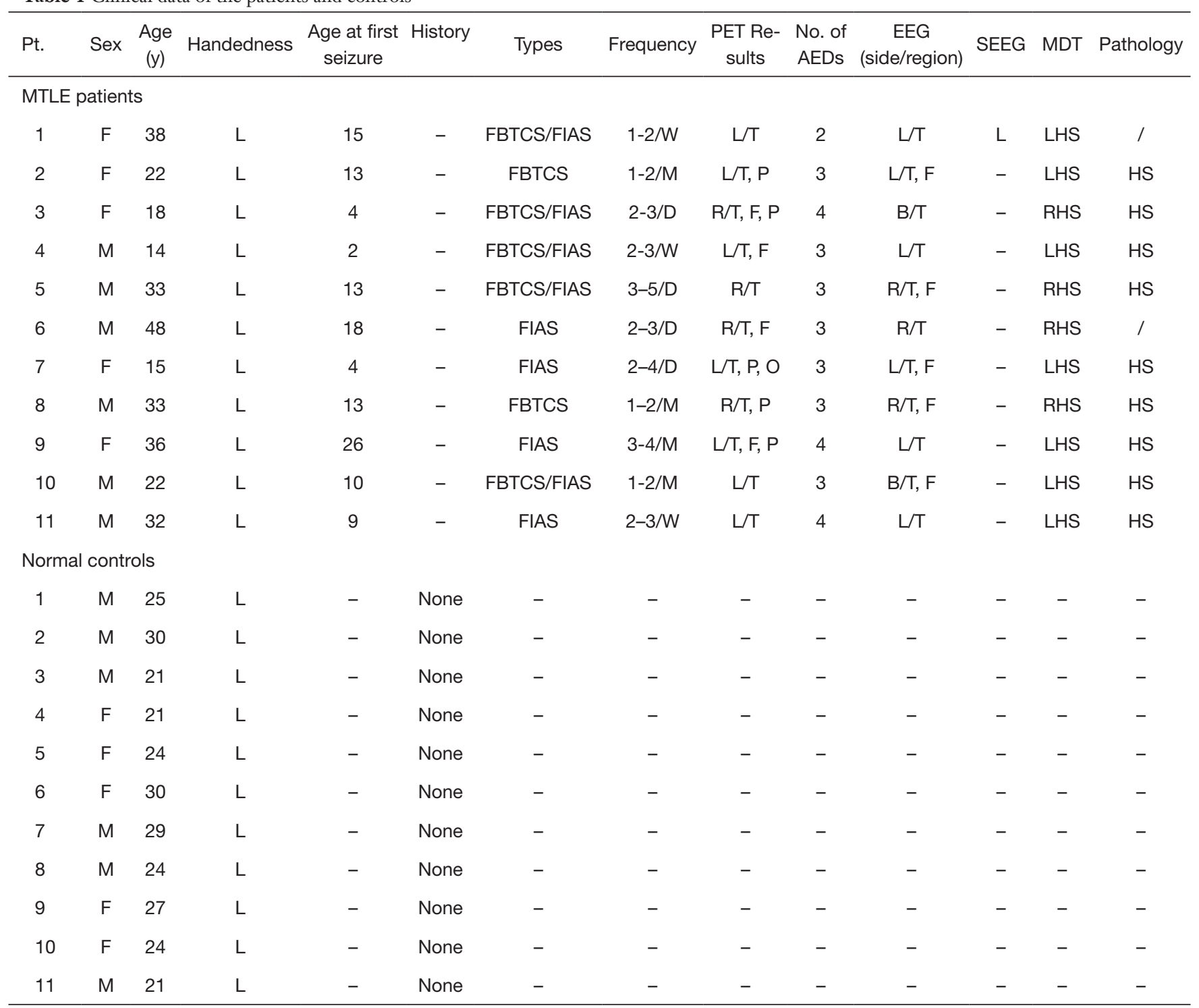

MTLE, mesial temporal lobe epilepsy; M, male; F, female; L, left; R, right; FBTCS, focal to bilateral tonic-clonic seizure; FIAS, focal impaired awareness seizure; B, bilateral; T, temporal area; F, frontal area; P, parietal area; O, occipital area; W, week; M, month; D, day; HS, hippocampal sclerosis. The side and the area of the EEG region were determined by the interictal scalp EEG.

using the same scanner (GE SIGNA Premier, General Electric Healthcare, Waukesha, WI, USA) at Xuanwu Hospital.

The3D MRI (T1-Weighted 3D magnetization prepared rapid acquisition gradient echo sequence, T1W1-3DMPRAGE) scanning parameters and conditions were as follows: slice thickness $=1 \mathrm{~mm}$; repetition time $(\mathrm{TR})=2,420 \mathrm{~ms}$; echo time $(\mathrm{TE})=2.376 \mathrm{~ms}$; inversion time $=900 \mathrm{~ms}$; field of view $=512 \mathrm{~mm} \times 512 \mathrm{~mm}$; number of excitations $=1$, and acquisition time $=6 \mathrm{~min}$.
The DSI scanning (half-q-space DSI with 256 directions) parameters and conditions were as follows: 64 channel head coils, b values range from 0 to $7,000 \mathrm{~s} / \mathrm{mm}^{2}$; TR $=5,548 \mathrm{~ms}$; $\mathrm{TE}=84.1 \mathrm{~ms}$; voxel size $=2 \mathrm{~mm} \times 2 \mathrm{~mm} \times 2 \mathrm{~mm}$; layer thickness $=2 \mathrm{~mm}$; imaging field $=112 \mathrm{~mm} \times 112 \mathrm{~mm}$, flip angle $=90^{\circ}$; parallel acquisition acceleration value $=2$; phase coding acceleration value $=3$; and acquisition time $=26 \mathrm{~min}$. The data processing and quality control were assessed by two experienced radiologists, while ensuring no head motion or other processing errors. 


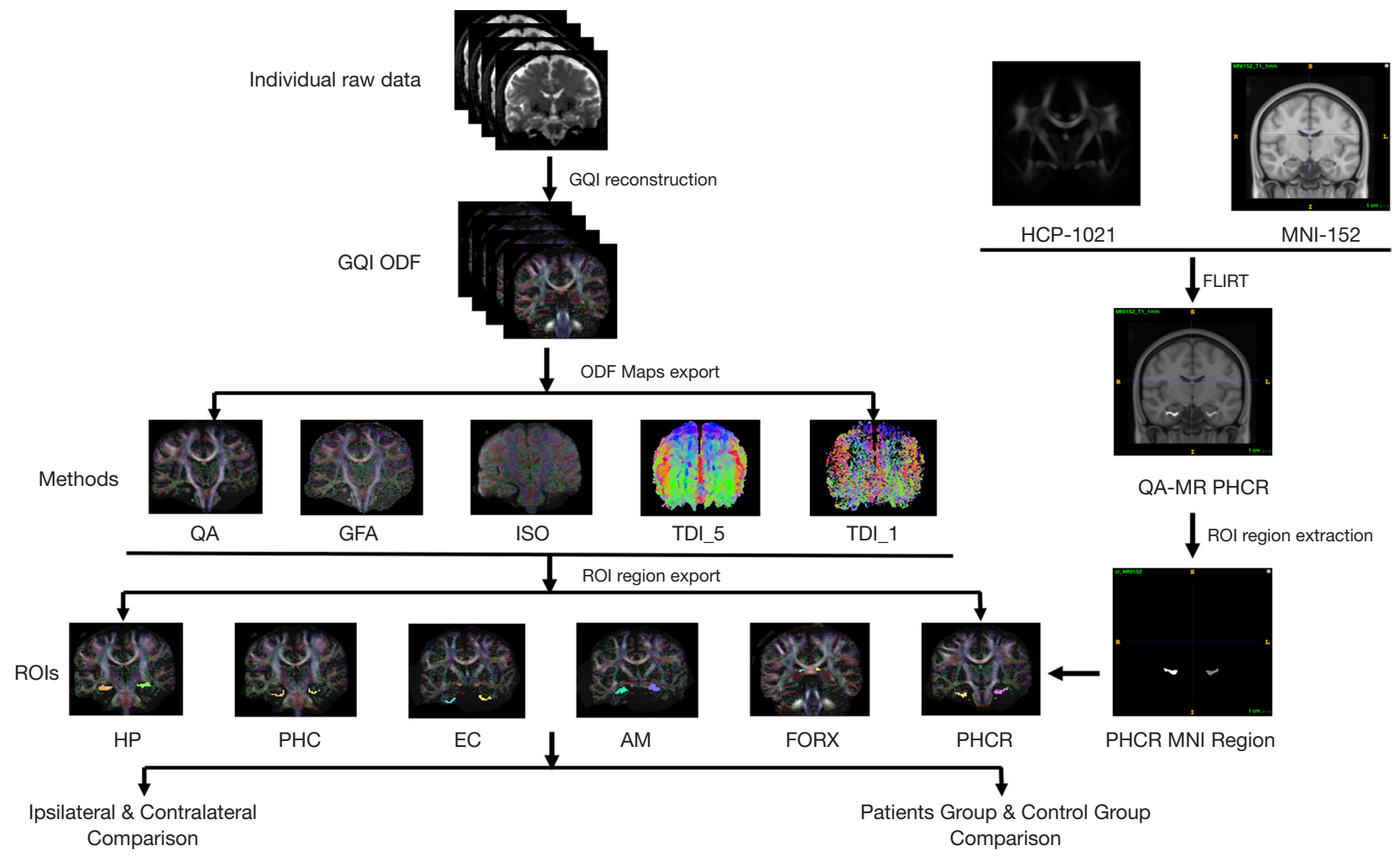

Figure 1 Raw data pre-processing flow chart. All raw data collections followed the same workflow. This chart demonstrates the process of diffusion scalar derivation, region of interest (ROI) exportation and quantitative analysis. Orientation distribution functions (ODF) maps for the 22 individuals were first reconstructed following the generalized q-sampling imaging (GQI) algorithm. ROIs were exported from DSIstudio followed by the quantitative comparison.

\section{Raw data pre-processing}

All data reconstruction was performed with DSIStudio software (http://dsi-studio.labsolver.org). Postreconstruction quality check was performed using the DSIStudio software to confirm the absence of registration error $(\mathrm{R}>0.6)$. Each voxel and the orientation distribution functions (ODF) were extracted with the generalized q-sampling imaging (GQI) algorithm for individual comparison (12). The reconstruction parameters for reconstruction were as follows: CSF calibration selected, diffusion sampling length ratio: 1.10; ODF tessellation: 20 folds; and number of fibers resolved: 10. A total of 22 individual representations of the ODF map in GQI space were created using the quantitative anisotropy (QA) and isotropic (ISO) values of each individual DSI data (Figure 1).

Track density imaging (TDI) value was another scalar in this study. It provides a relatively novel and quantitative method to produce high-quality white matter images with high spatial resolution (13-15). For each TDI reconstruction, the individual tracking threshold was set at $60 \%$ of the maximum value with MATLAB R2018a (Mathworks, Natick, Mass, USA). Whole brain seeding was processed with an angular threshold of $90^{\circ}$. For gray matter, the step size was 0.1 , minimum (Min) length $=0 \mathrm{~mm}$, and maximum (Max) length $=5 \mathrm{~mm}$. For white matter fiber bundles, the step size was 0.5 , Min length $=5 \mathrm{~mm}$, Max length $=300 \mathrm{~mm}$, the fiber tracking stopped when the maximum number of seeds was 2,000,000 (Figure 1).

\section{Region of interest (ROI) selection and extraction}

In this study, the bilateral hippocampus (HP), parahippocampus (PHC), amygdala (AM), and entorhinal cortex (EC) were the main gray matter structures examined, and for white matter, the crus of fornix (FORX) and the parahippocampal radiation of the cingulum (PHCR) were selected to compare the heterogeneity of the examined 
scalars between the two sides of MTLE patients and controls.

For HP, PHC, AM, and EC, the ROI in GQI space can be exported and saved from the embedded template in DSI studio software. As for bilateral FORX, fiber tracking and reconstruction methods were used, and calculated region was restricted only to the crus of the bilateral fornix. The tracking parameters were as follows: step size $=0.1$, angular threshold $=90^{\circ}$, Min length $=5 \mathrm{~mm}$, and Max length $=300 \mathrm{~mm}$. The fiber tracking stopped when the maximum number of seeds was 50,000. The FORX in GQI space were then exported (Figure 1).

For PHCR, the module of segmentation editor used was 3D Slicer software (https://www.slicer.org). The QA map in the Montreal Neurological Institute (MNI) region was first created using Functional Magnetic Resonance Imaging of the Brain(FMIRB) Linear Image Registration Tool (FLIRT) in FSL (FMRIB Software Library) combining the MNI152 (http://nist.mni.mcgill.ca/?page_id=714) template and the HCP1201 (http://brain.labsolver.org/diffusion-mritemplates/hcp-842-hcp-1021) template. The diffusion MR map in MNI space was then used in 3D Slicer to restrict the threshold with only the white matter visible (no gray matter). The visualized white matter regions adjacent to the PHC were extracted as PHCR with a comparable length to the PHC. Finally, the bilateral PHCR in the MNI space was imported to DSI-Studio software and exported as ROIs in GQI space (Figure 1).

\section{Quantitative analysis of ROIs}

As the patients included herein had a definitive diagnosis of MTLE, the capability of FORX and PHCR to determine lateralization was assessed in our study. The bilateral QA, ISO and TDI values of the FORX and PHCR were compared between the ipsilateral side (the side with MTLE) and the contralateral side using Paired $t$-test or the Wilcoxon test if the data set did not fit the normal distribution. The comparison between the two ROIs were further investigated among the ipsilateral side, contralateral side, and the controls separately. Finally, in order to observe whether FORX_TDI and PHCR_TDI can be lateralized for MTLE, a descriptive analysis of the sensitivity and specificity between the two structures were compared in lateralization of MTLE using receiver operating characteristic (ROC) curve.

The quantitative analysis for gray matter structures was conducted between the HP, PHC, AM and EC. The heterogeneity between the bilateral mesial temporal areas in patients with MTLE were first processed. The four ipsilateral ROIs were compared to each other and $\mathrm{P}<0.05$ was considered statistically significant. The bilateral side of the four ROIs were compared separately with the control data collected from the volunteers. Specificity was calculated between them with the Independent-samples $t$-test or the Mann-Whitney U test. Significant level $\alpha(\alpha=0.05)$ was adjusted as 0.017 using Bonferroni method in order to avoid Type I error when performed multiple comparisons of QA and ISO among ipsilateral and contralateral values, and controls. TDI values were only compared between ipsilateral and contralateral regions, thus the level $\alpha$ was determined as 0.05 .

\section{Statistical analysis}

All statistical analyses were performed with the Solutions Statistical Package for the Social Sciences (SPSS, Version 22; IBM, Armonk, New York, USA). The figures in our study were editing and drawing using Photoshop (Version CC 2019 for Mac, Adobe Systems Incorporated, San Jose, USA) and GraphPad Prism (Version 8.0 for Mac, GraphPad Software, San Diego, USA).

\section{Results}

\section{General information}

In this study, we recruited 11 patients ( 6 men and 5 women, age: $28.3 \pm 10.8$ years) with unilateral medial temporal lobe epilepsy (7 left and 4 right) from the Department of Neurosurgery in Xuanwu Hospital between May 2019 and January 2020. In these 11 patients, we observed a series of abnormal discharges during the interictal phase. Most of the patients have a lateralization on the scalp-EEG results with some of the patients showing bilateral asynchronous discharges during the ictal and interictal period (Table 1 and Figure 2). All the patients had a definite diagnosis of MTLE after intensive discussion by the MDT consisting of a neurosurgeon, a neurologist, and a radiologist. The seizure type of the 11 patients were listed according to the 2017 ILAE classification, all the patients suffered from the focal onset with FBTCS (focal to bilateral tonic-clonic seizure) and/or FIAS (focal impaired awareness seizure) (16).

Among the 11 patients included, nine underwent a standard ATL with a clear pathology diagnosis of HP sclerosis (Table 1). One patient underwent the implantation 

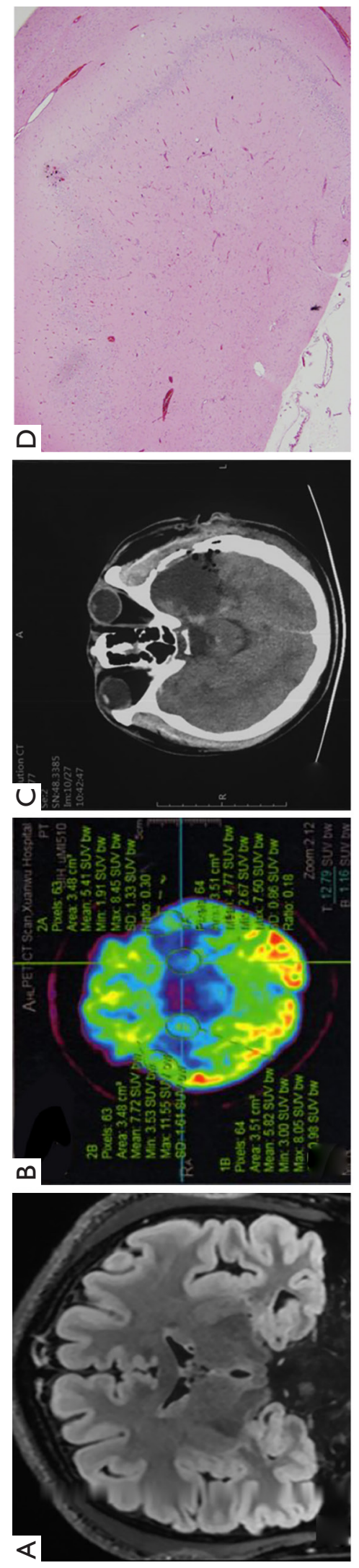

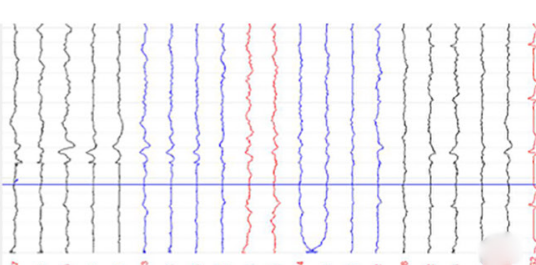

○$$
\text { }
$$
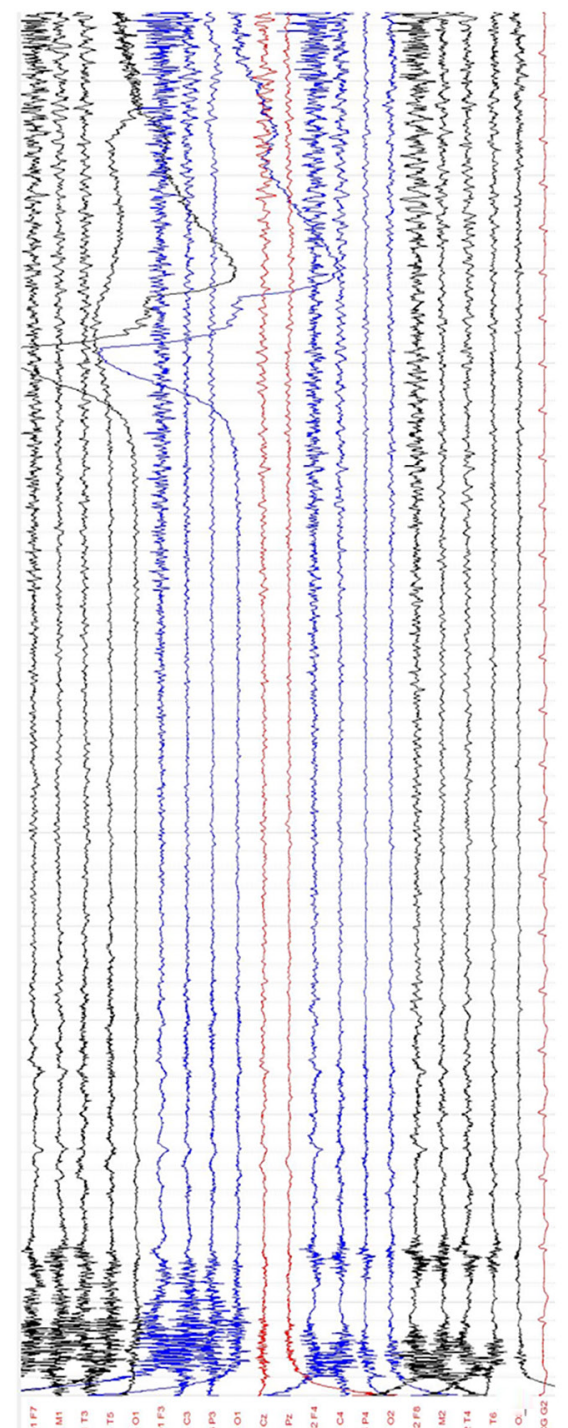

4

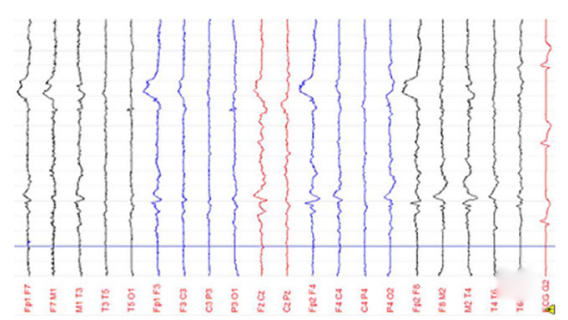

ш

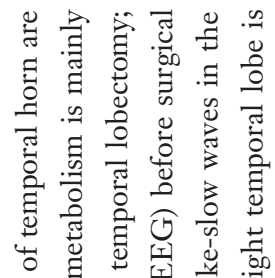

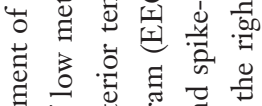

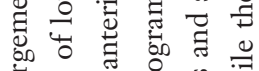

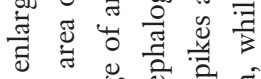

范

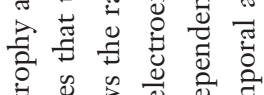

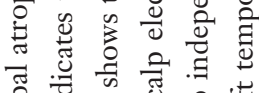

范

厄ّٓ

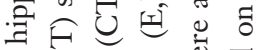

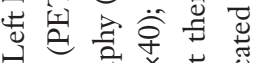

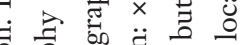

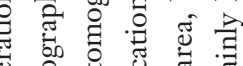

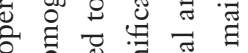

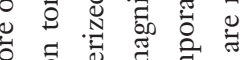

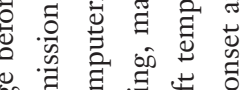

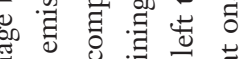

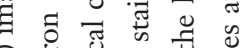

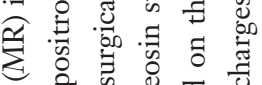

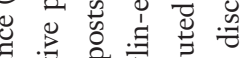

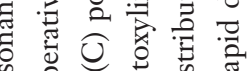

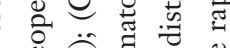

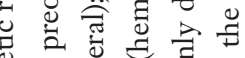

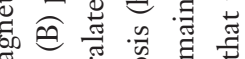

is

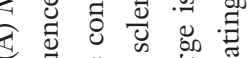

$\therefore$ 萬

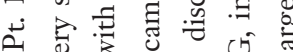

E 3 .

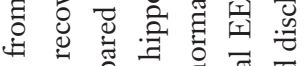

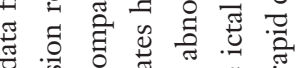

5

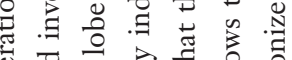

एँ ज

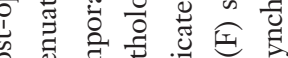

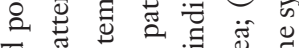

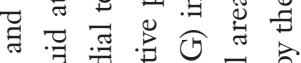

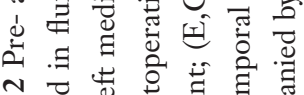

フ

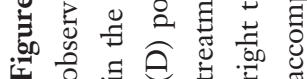


Table 2 Statistics results between ipsilateral and contralateral sides

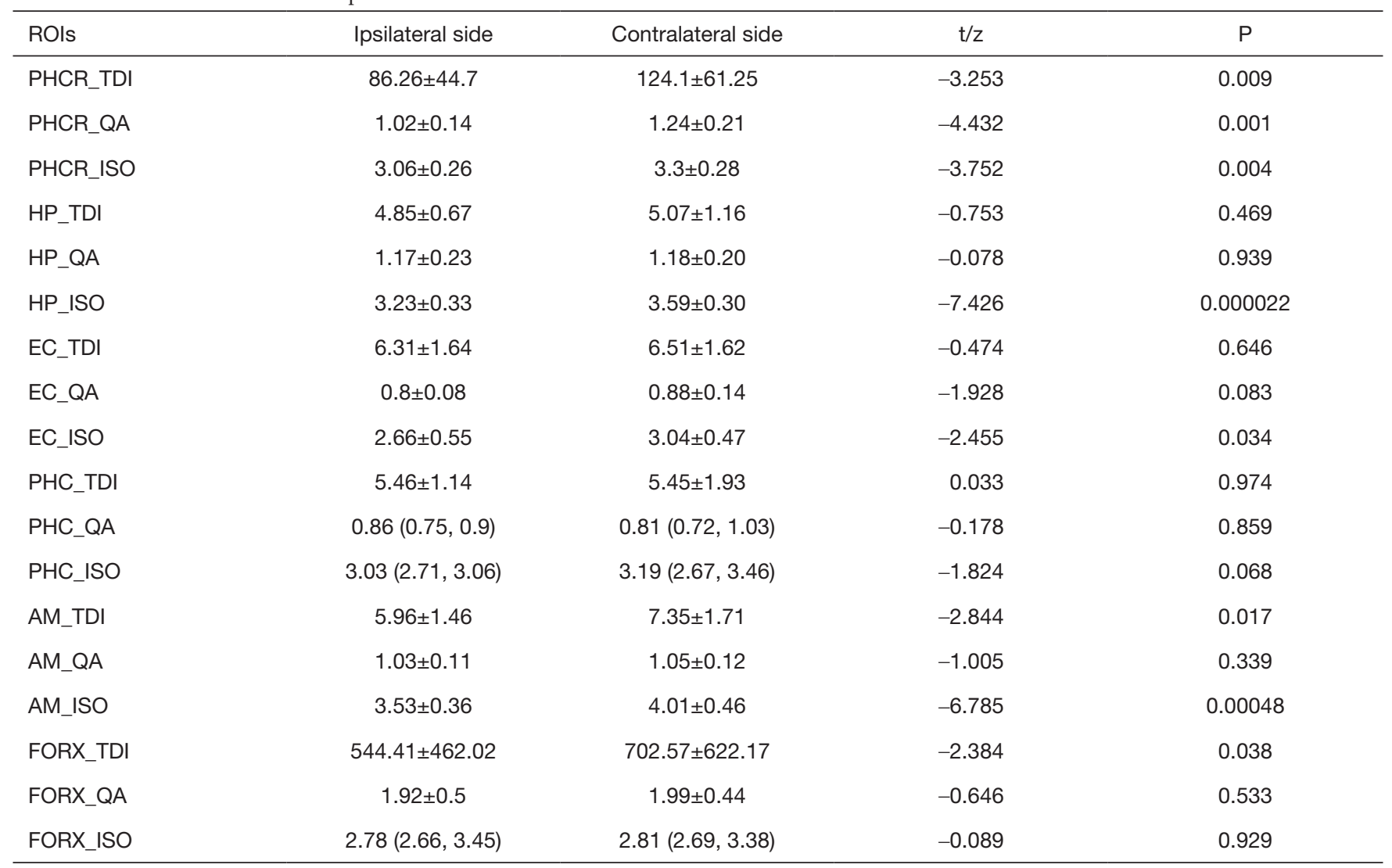

Paired $t$-test or Wilcoxon test was used between ipsilateral and contralateral sides (PHC_QA, PHC_ISO, FORX_ISO were analyzed using Wilcoxon test). PHCR, parahippocampal radiation of the cingulum; TDI, track density imaging; QA, quantitative anisotropy; FORX, fornix.

of frameless SEEG electrodes (Alcis, Besancon, France) and long-term intracranial EEG recording with definite SEEG results. Only one patient had no invasive treatment following a clear diagnosis of MTLE after MDT discussion.

Meanwhile, 11 healthy volunteers ( 6 men and 5 women, age: $25.1 \pm 3.5)$ were recruited and became the control group. There were no significant differences in age and sex between patients and controls $(\mathrm{P}=0.371$ and 1.00$)$.

\section{Comparison between the ROIs in patients and controls}

For PHCR and FORX, the quantitative analysis was first processed between the ipsilateral and contralateral sides of the patients. Our results showed that, for PHCR, the value of QA, ISO and TDI values were significantly decreased than those of the contralateral side $(\mathrm{P}=0.001,0.004,0.009$, respectively, Paired $t$-test, Bonferroni correction). As for the FORX, the value of TDI was significantly decreased $(\mathrm{P}=0.038$, Paired $t$-test, Bonferroni correction) with another two scalars showing no difference between the two sides (Paired $t$-test for QA values and Wilco xon for ISO values, $\mathrm{P}>0.05)$. The results are displayed on Table 2 . Next, the comparison between the patients and controls was performed. Since TDI determination was performed with a different threshold when fiber tracking and the error cannot be eliminated when comparing patients and controls (the individual tracking threshold was set at $60 \%$ of the maximum value), the QA and ISO were selected to finish the comparison. Results showed that the difference in ipsilateral PHCR_QA and PHCR_ISO in the patients were greatly significant compared with the volunteers (Independent-Sample $t$-test, $\mathrm{P}<0.05$ ), while PHCR_QA still showing significant difference after correction for multiple comparisons $(\mathrm{P}<0.017)$. When comparing the contralateral side with the control groups, there were no differences in FORX and PHCR (Table 3 and Table 4). Additionally, the reconstruction of cingulum and fornix are shown as QA values on Figure 3 and Figure 4. 
Table 3 Statistical results between ipsilateral side and control group

\begin{tabular}{lcccc}
\hline ROIs & Ipsilateral side & Control group & t/z & P \\
\hline PHCR_QA & $1.02 \pm 0.14$ & $1.23 \pm 0.19$ & -3.291 & 0.002 \\
PHCR_ISO & $3.06 \pm 0.26$ & $3.28 \pm 0.28$ & -2.261 & 0.031 \\
HP_QA & $1.17 \pm 0.23$ & $1.23 \pm 0.19$ & -0.701 & 0.488 \\
HP_ISO & $3.23 \pm 0.33$ & $3.54 \pm 0.32$ & -2.634 & 0.013 \\
EC_QA & $0.8 \pm 0.08$ & $0.93 \pm 0.15$ & -2.615 & 0.014 \\
EC_ISO & $2.66 \pm 0.55$ & $2.99 \pm 0.55$ & -1.602 & 0.119 \\
PHC_QA & $0.86(0.75,0.9)$ & $0.92(0.81,1.18)$ & -1.893 & 0.058 \\
PHC_ISO & $3.03(2.71,3.06)$ & $3.11(2.96,3.4)$ & -2.083 & 0.037 \\
AM_QA & $1.03 \pm 0.11$ & $1.11 \pm 0.15$ & -1.655 & 0.108 \\
AM_ISO & $3.53 \pm 0.36$ & $3.85 \pm 0.48$ & -1.901 & 0.067 \\
FORX_QA & $1.92 \pm 0.5$ & $2.10 \pm 0.21$ & -1.17 & 0.265 \\
FORX_ISO & $2.78(2.66,3.45)$ & $2.83(2.74,3.12)$ & -0.382 & 0.702 \\
\hline
\end{tabular}

Independent-samples $t$-test or the Mann-Whitney $U$ test was used between ipsilateral side and control group (PHC_QA, PHC_ISO and FORX_ISO were calculated using Mann-Whitney U test). PHCR, parahippocampal radiation of the cingulum; QA, quantitative anisotropy; ISO, isotropic; HP, hippocampus; EC, entorhinal cortex; PHC, parahippocampus; AM, amygdala; FORX, fornix.

Table 4 Statistical results between contralateral side and control group

\begin{tabular}{|c|c|c|c|c|}
\hline ROls & Contralateral side & Control group & $t / z$ & $\mathrm{P}$ \\
\hline PHCR_ISO & $3.3 \pm 0.28$ & $3.28 \pm 0.28$ & 0.123 & 0.903 \\
\hline HP_QA & $1.18 \pm 0.20$ & $1.23 \pm 0.19$ & -0.669 & 0.508 \\
\hline HP_ISO & $3.59 \pm 0.30$ & $3.54 \pm 0.32$ & 0.446 & 0.659 \\
\hline EC_ISO & $3.04 \pm 0.47$ & $2.99 \pm 0.55$ & 0.294 & 0.771 \\
\hline PHC_QA & $0.81(0.72,1.03)$ & $0.92(0.81,1.18)$ & -1.739 & 0.082 \\
\hline PHC_ISO & $3.19(2.67,3.46)$ & $3.11(2.96,3.4)$ & -0.325 & 0.745 \\
\hline AM_QA & $1.05 \pm 0.12$ & $1.11 \pm 0.15$ & -1.08 & 0.288 \\
\hline FORX_ISO & $2.81(2.69,3.38)$ & $2.83(2.74,3.12)$ & -0.401 & 0.688 \\
\hline
\end{tabular}

Independent-samples $t$-test or the Mann-Whitney $U$ test was used between ipsilateral side and control group (PHC_QA, PHC_ISO and FORX_ISO were calculated using Mann-Whitney U test). PHCR, parahippocampal radiation of the cingulum; QA, quantitative anisotropy; ISO, isotropic; HP, hippocampus; EC, entorhinal cortex; PHC, parahippocampus; AM, amygdala; FORX, fornix. 


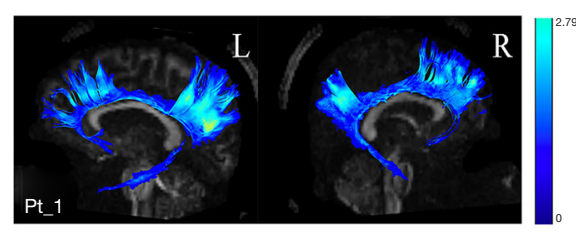

$q a=0.94$ $\mathrm{qa}=1.22$

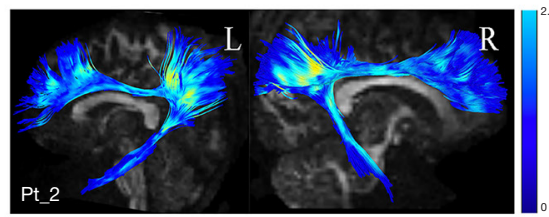

$q a=1.67$

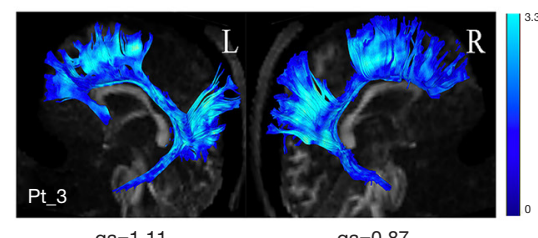

$q a=1.11$ $q a=0.87$

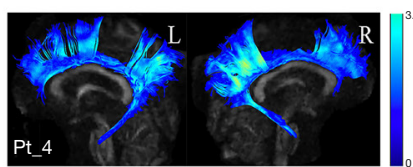

$q a=0.80$

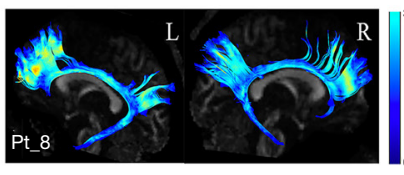

$q a=1.17$

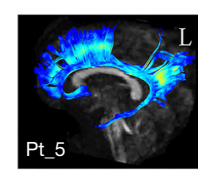

$q a=1.38$

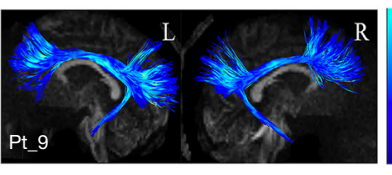

$q a=1.01$

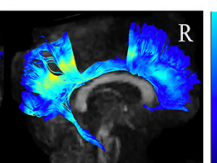

$q a=1.09$

$\mathrm{qa}=1.12$

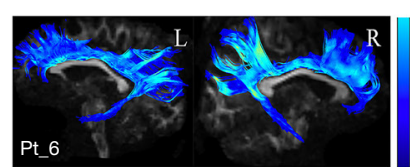

$q a=1.23$

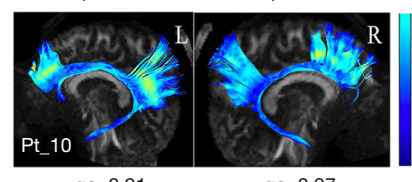

$q a=0.91$

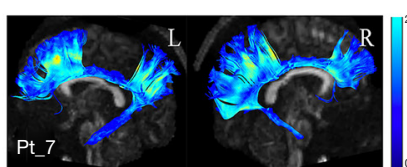

$q a=1.08$ $q a=1.28$

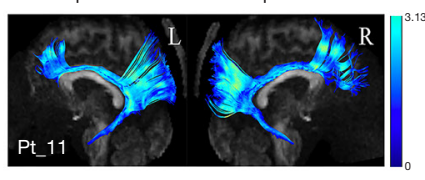

$q a=0.96$ $q a=0.92$

Figure 3 Bilateral cingulum and quantitative anisotropy (QA) visualization for 11 patients. The bilateral cingulum is reconstructed and quantitatively analyzed. The QA value is visibly referenced by the color bar on DSI-Studio. The QA value on the ipsilateral side on the parahippocampal radiation of the cingulum (PHCR) area is significantly decreased.

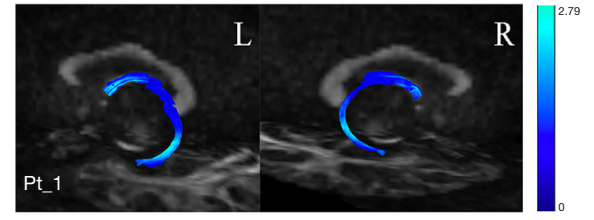

$\mathrm{qa}=1.15$

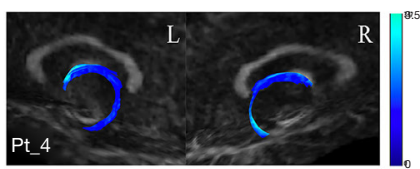

$q a=1.20$

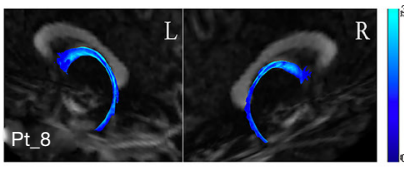

qa $=1.95$

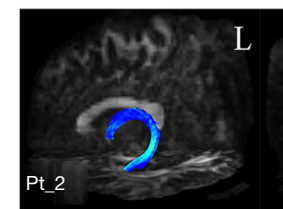

$\mathrm{qa}=2.93$

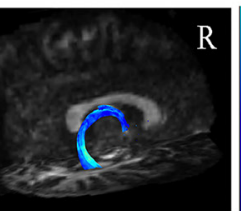

$q a=2.48$

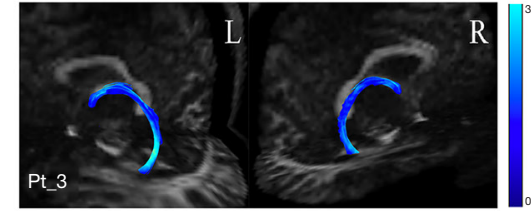

$q a=2.18$

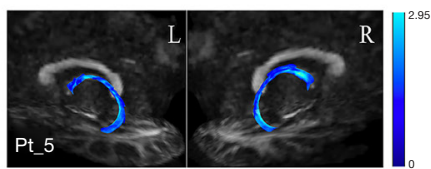

$q a=1.66$

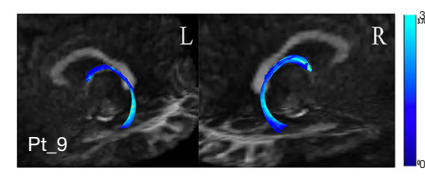

$\mathrm{qa}=1.79 \quad \mathrm{qa}=2.24$

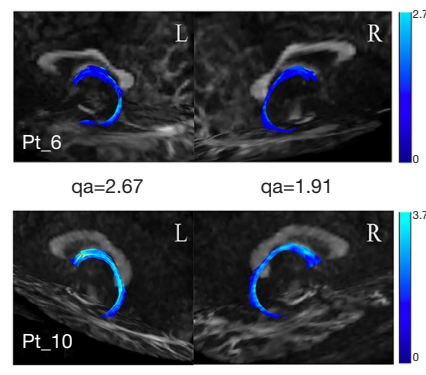

$q a=2.17$

$\mathrm{qa}=1.68$

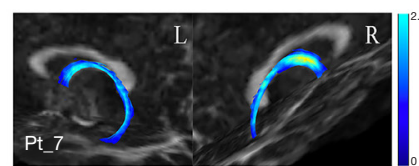

$q a=2.10 \quad q a=2.15$

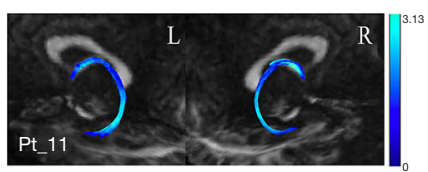

$q a=2.20 \quad q a=2.22$

Figure 4 Bilateral fornix and quantitative anisotropy (QA) visualization for 11 patients. The fornix is reconstructed and quantitatively analyzed following the study protocol. The QA value is visibly referenced by the color bar on DSI-Studio. The QA value on the ipsilateral side of the crus of fornix (FORX) area is significantly decreased.

As both FORX_TDI and PHCR_TDI measurements showed significantly decreased values in the ipsilateral side, which was better in finding the asymmetry than QA and ISO values, the comparison of sensitivity and specificity in detecting the lateralization of MTLE between FORX_ TDI and PHCR_TDI was conducted with ROC curve for both patients and controls. There were $9 / 11$ patients whose pathologic findings were consistent with $\mathrm{PHCR}_{-} \mathrm{TDI}$ abnormalities, and 0/11 healthy volunteers had PHCR_TDI abnormalities, which indicated the sensitivity was $89.5 \%$ and specificity $100.0 \%$ for PHCR_TDI (area under curve $=0.93, \mathrm{P}=0.019$; Table 5, Figure 5). Meanwhile, there were $10 / 11$ patients whose pathologic findings were consistent with FORX_TDI abnormalities, and 0/11 healthy 
volunteers had FORX_TDI abnormalities, which indicated the sensitivity was $95.0 \%$ with the specificity $100 \%$ for FORX (area under curve $=0.95, \mathrm{P}=0.040$, Table 5, Figure 5). As a quantitative diffusion modality, DSI may have some advantages in finding heterogeneity between white matters. In this study, ROC curves were used to assess effectiveness of TDI scalar and comparing the sensitivity between PHCR_TDI and FORX_TDI. Our results showed that these two white matter structures in TDI can effectively be used to detect the lateralization of MTLE. Moreover, FORX_TDI appeared more sensitive than PHCR_TDI.

For the gray matter, as a matter of priority, the values of bilateral HP, PHC, AM, and EC were compared to

Table 5 ROC analysis

\begin{tabular}{|c|c|c|c|c|}
\hline \multirow{2}{*}{ AUC } & \multirow{2}{*}{$\begin{array}{l}\text { Standard } \\
\text { error }\end{array}$} & \multirow{2}{*}{$P$} & \multicolumn{2}{|c|}{$95 \%$ confidence interval } \\
\hline & & & Min & Max \\
\hline \multicolumn{5}{|c|}{ Test variable: PHCR_TDI } \\
\hline 0.930 & 0.058 & 0.019 & 0.816 & 1.000 \\
\hline \multicolumn{5}{|c|}{ Test variable: FORX_TDI } \\
\hline 0.950 & 0.049 & 0.040 & 0.854 & 1.000 \\
\hline
\end{tabular}

ROC, receiver operating characteristic; PHCR, parahippocampal radiation of the cingulum; TDI, Track density imaging; FORX, fornix.

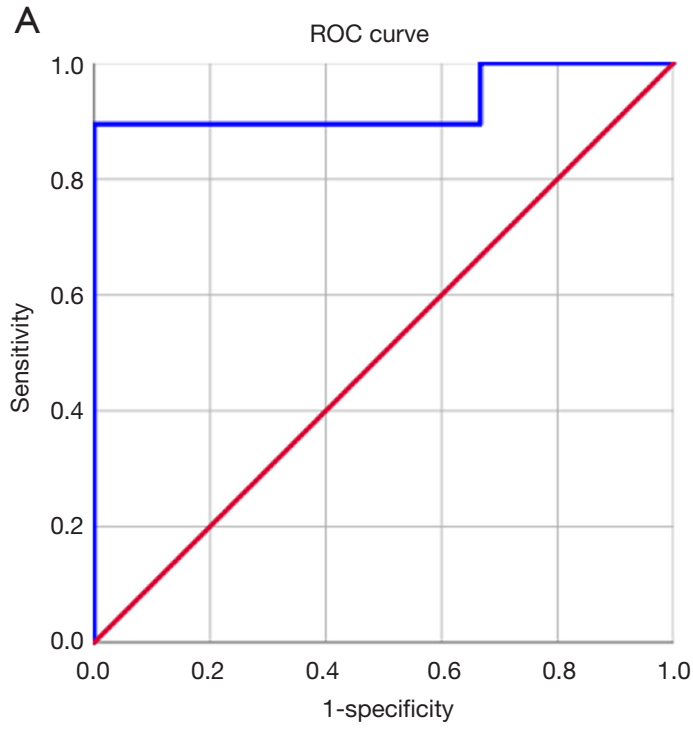

assess the diffusion heterogeneity between the ipsilateral and contralateral mesial temporal area. Our results showed that the values of HP_ISO, EC_ISO, AM_TDI, and AM_ ISO were significantly different on the contralateral side (Paired $t$-test, $\mathrm{P}<0.05$ ). Moreover, HP_ISO still survived after the Bonferroni method correction $(\mathrm{P}<0.017)$ with the rest of the values showing a decreasing trend with no statistically significant difference (Table 2). The values of bilateral HP, PHC, AM and EC between the ipsilateral and contralateral sides and the controls were compared to further assess the heterogeneity. At first, the ipsilateral side of the four ROIs were compared with those of the control group. The results showed that the value of HP ISO, EC_QA, and PHC_ISO were significantly decreased on the ipsilateral side (Independent-Sample $t$-test for HP ISO and EC_QA, Mann-Whitney U test for PHC_ISO, $\mathrm{P}<0.05)$. After the correction, HP_ISO and EC_QA showed significantly difference with the other regions showing no differences between groups $(\mathrm{P}<0.017$, Table 3). Moreover, the contralateral side of the MTLE patients showed no difference when compared to that of the healthy controls (Table 4). All the statistical results can be seen in Figure 6.

\section{Discussion}

In our study, we quantitatively demonstrated the

B

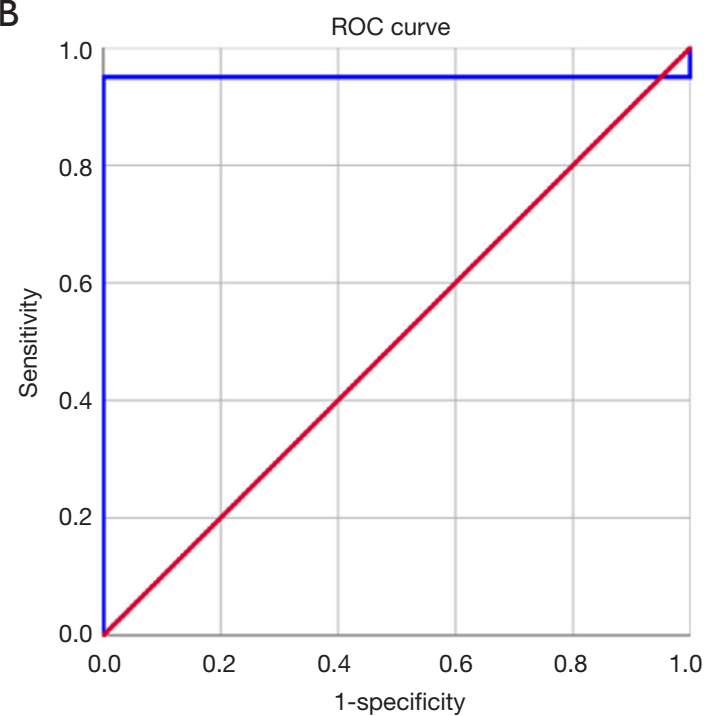

Figure 5 ROC curve for PHCR_TDI and FORX_TDI. (A) The ROC curve for PHCR_TDI shows that the area under curve was 0.93. (B) The area under curve for FORX_TDI is 0.95 , which is higher than PHCR_TDI. PHCR, parahippocampal radiation of the cingulum; ROC, receiver operating characteristic; TDI, Track density imaging. 

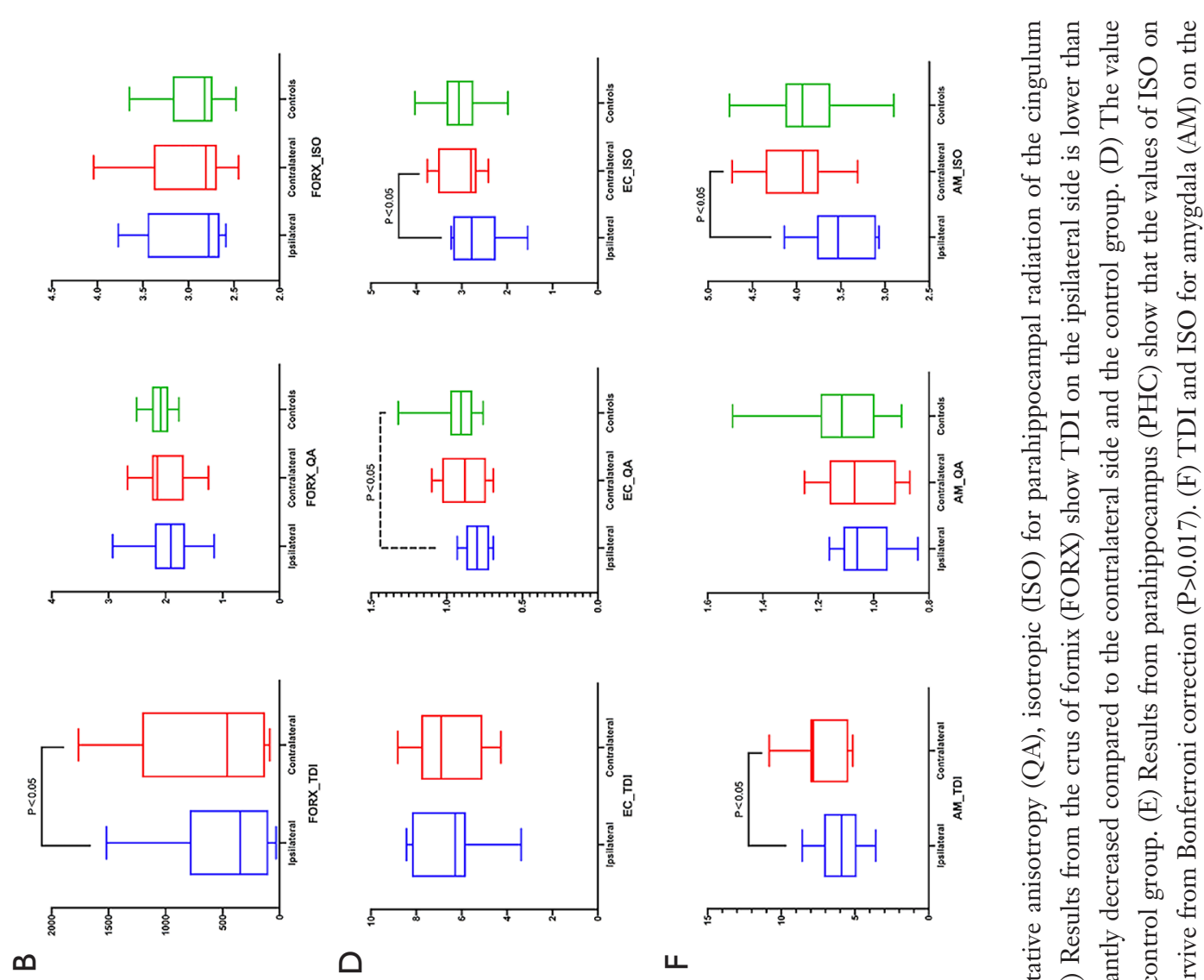

レ
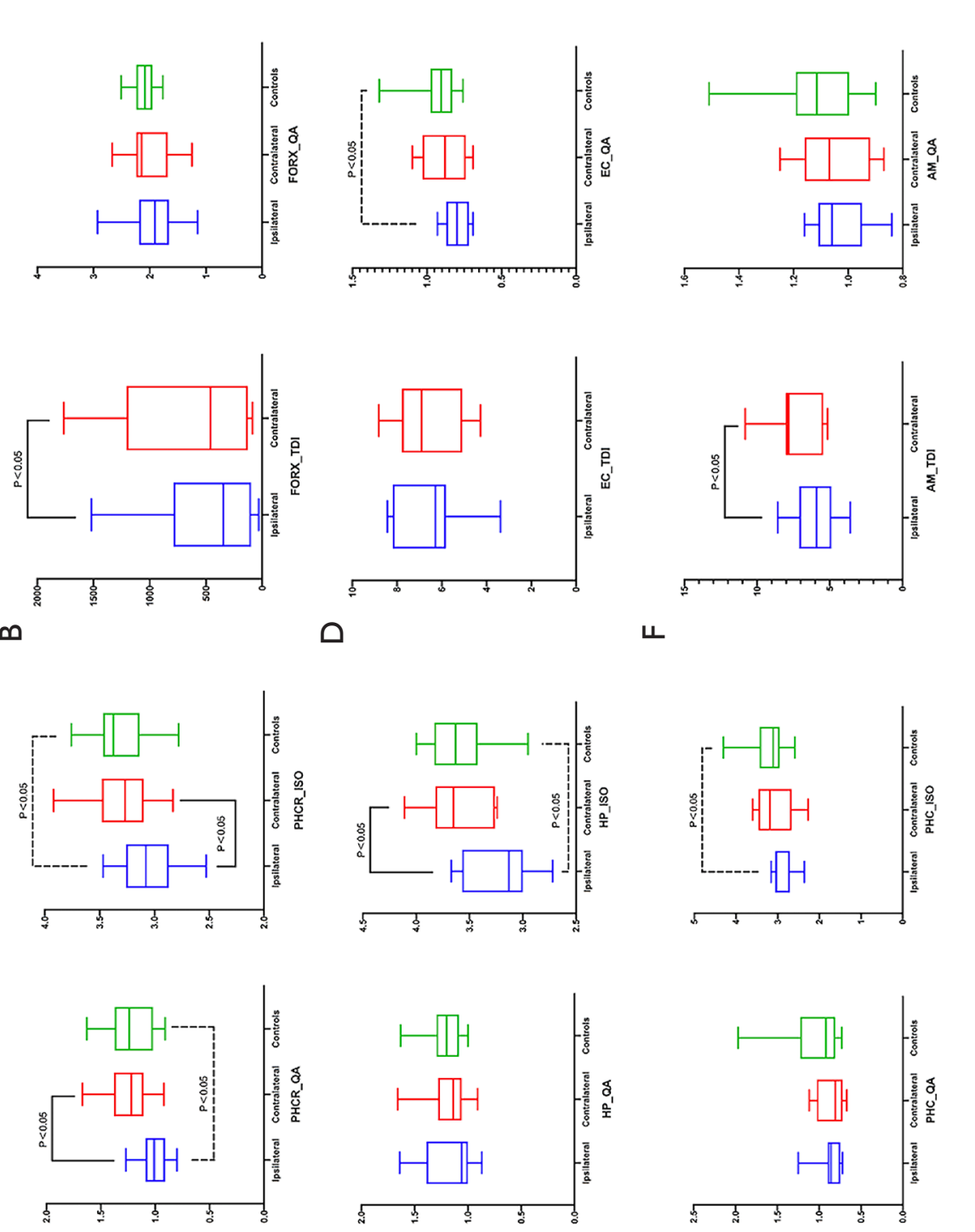

\section{产.} ㄴ.

$\therefore$ 虫

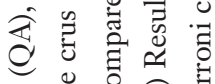

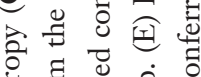

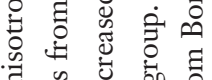

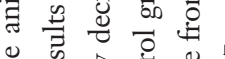

.

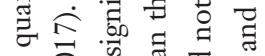

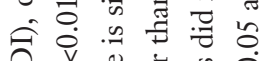

Ev v v

so

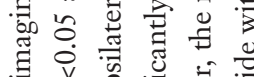

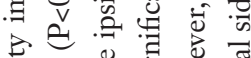

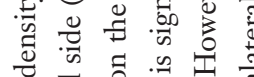

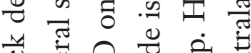

苛焉

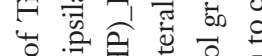

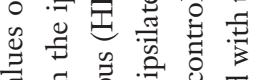

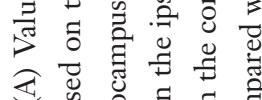

ङ

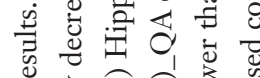

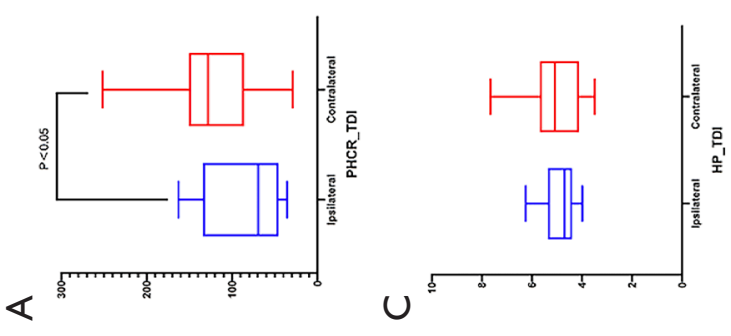

山

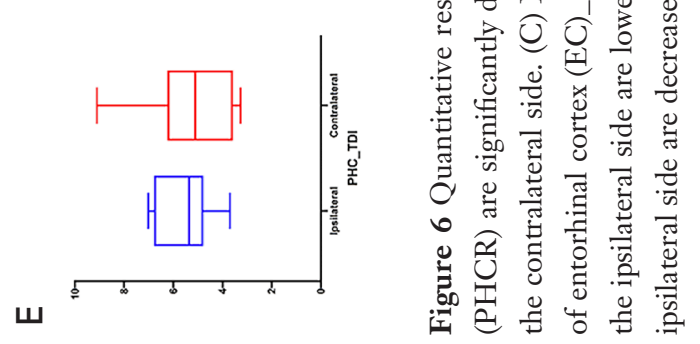


heterogeneity on the diffusion level in patients with MTLE using DSI. To the best of our knowledge, this is the first report using the GQI algorithm in DSI to quantify the diffusion differentiation between the two sides of unilateral MTLE. Based on our results, we concluded a decreased level of diffusion existed on some of the mesial temporal structures. As a preliminary exploration, DSI, with its high angular resolution may be a reliable and effective modality for the presurgical evaluation of MTLE patients.

\section{PHCR and FORX-important input and output from the $H P$}

In our 11 unilateral MTLE patients, significant differences were found on the bilateral PHCR and FORX. A series of decreased values were found on the ipsilateral side of the MTLE patients. The cingulum and fornix are the two important parts of the Papez circuit (17). Within the circuit, the cingulum bundle forms a collection of fibers that project from the cingulate gyrus to the EC and $\mathrm{PHC}$, and this connection is reported to be associated with a series of functions such as emotion, reward, pain and memory $(6,18)$. In contrast, the fornix forms a connection with the HP and the mammillary bodies, mainly affecting the major function of memory (19). As hippocampal sclerosis is the major pathological change in MTLE, microstructural changes in relation to the HP may occur due to the repetitive seizure attacks (1).

\section{High resolution diffusion scalar of the FROX and PHCR}

Studies focusing on cingulum and fornix have found the diffusion differences. Reports have stated the presence of widespread white and gray matter abnormalities in patients with TLE (2). Urbach et al. reported a reduction in the bilateral cingulum fiber in unilateral temporal lobe epilepsy with HS (6). To the best of our knowledge, most of the previously reported studies have used the technique of DTI to quantify the changes, which may lead to inaccuracy results as it only decodes the average trend of the orientation of fibers for each voxel. Lemkaddem $e t$ al. (2014) has used high angular resolution DSI to investigate the microstructural alterations in TLE. They found that the network topology of the unilateral TLE was less efficient to detect structural connectivity in patients compared to the control group from the aspect of structural connectivity based on the anisotropy of the diffusion (3). However, they failed to assess the diffusion heterogeneity between the two sides in MTLE patients with the help of DSI in GQI space. Besides QA and ISO using GQI algorithm, TDI has been the preferred quantitative image reconstruction method of choice for dMRI to provide spatial resolution and anatomical information, which cannot be obtained by routine methods, especially for the white matter $(14,15)$. In this study, we observed a significantly decreased diffusion level in the PHCR and FORX, corresponded with the microstructural changes for the white matter of MTLE patients. Moreover, the sensitivity and specificity for PHCR_TDI and FORX_TDI values were compared between the two structure in detecting the lateralization of MTLE. Our results showed that both ROIs in TDI had $100 \%$ specificity and higher sensitivity $(89.5 \%$ and $95 \%$, respectively) to detect the ipsilateral lateralization of MTLE. FORX in TDI tended to be more sensitive than PHCR in TDI (AUC $=0.93 v s$. $\mathrm{AUC}=0.95$ ).

\section{Diffusion changes of the other major structures}

The diffusion level between the different subcortical structures on the bilateral side has also been quantified with DSI. As tractography is a useful method for presurgical evaluation, itis being increasingly used to investigate epilepsy, specifically TLE $(4,7,20)$. Studies have found that on DTI, the subcortical structures, including the amygdala, HP and thalamus, ipsilateral to the seizure side, have low fractional anisotropy values (2,21-23). As DSI can resolve intravoxel diffusion heterogeneity by measuring its diffusion density spectra estimator, it tends to be more precise than DTI methods in the quantification of the diffusion level $(9,24)$. However, no study has assessed the diffusion changes between mesial temporal gray matter structures using DSI in patients with MTLE. In our study, the diffusion level on different ROIs were significantly decreased including the $\mathrm{HP}, \mathrm{AM}$ and $\mathrm{EC}$ in $\mathrm{GQI}$ space $(\mathrm{P}<0.017)$. Our preliminary results showed that although diffusion may have some advantages in finding heterogeneity between white matters, there still existed some differences between subcortical structures like HP. The diffusion level may vary in MTLE patients for both white matter and grey matter and a further investigation may be needed from the aspect of both brain networks and connection omics.

\section{Limitations}

Limitations still exist in our present study. First, the sample size we evaluated was small. There may have been some 
selection bias for these data because of the small sample size. Moreover, as DSI is a newly developed imaging modality, the results presented herein are preliminary. As DSI will gradually be a routine method for both clinical and scientific use, a larger number of patients and controls may be required for further assessment. Second, in this study, we did not perform a comparison of the accuracy between DTI and DSI in lateralization of MTLE in this study. The conclusion that DSI has higher angular resolution was summarized from the previous study $(24,25)$. A further study on simultaneously acquired DTI and DSI scans may be performed if necessary. Finally, other parts of the ROIs such as the uncinate gyrus were not included in our comparisons and need to be further investigated in our future studies.

\section{Conclusions}

We have compared the diffusion differences between patients with MTLE and healthy controls using highly angular DSI with GQI algorithm in unilateral MTLE. We investigated the heterogeneity in both gray and white matters between the two sides and found that the quantitative diffusion scalars of the DSI are sufficiently sensitive to define the ipsilateral lateralization of MTLE during the presurgical evaluations. As a preliminary result, this quantitative technique may play a supplementary noninvasive role in presurgical assessment of MTLE patients, especially in cases when conventional MRI findings tends to be negative.

\section{Acknowledgments}

The authors want to thank all the volunteers who participated in this study. We also want to thank our colleagues from the Department of Radiology, Xuanwu Hospital, for offering the assistance with data processing. Funding: This work was supported by the National Natural Science Foundation of China (Grant Numbers 81801288, 81871009) and Green Seedling Plan, Beijing Municipal Administration of Hospitals (QML20190805).

\section{Footnote}

Reporting Checklist: The authors have completed the STROBE reporting checklist. Available at http://dx.doi. org/10.21037/atm-20-5719

Data Sharing Statement: Available at http://dx.doi. org/10.21037/atm-20-5719

Conflicts of Interest: All authors have completed the ICMJE uniform disclosure form (available at http://dx.doi. org/10.21037/atm-20-5719). The authors have no conflicts of interest to declare.

Ethical Statement: The authors are accountable for all aspects of the work in ensuring that questions related to the accuracy or integrity of any part of the work are appropriately investigated and resolved. The study was conducted in accordance with the Declaration of Helsinki (as revised in 2013). The study was approved by the ethics committee at Xuanwu Hospital (NO. LYS [2019] 097) and informed consent was taken from all the patients.

Open Access Statement: This is an Open Access article distributed in accordance with the Creative Commons Attribution-NonCommercial-NoDerivs 4.0 International License (CC BY-NC-ND 4.0), which permits the noncommercial replication and distribution of the article with the strict proviso that no changes or edits are made and the original work is properly cited (including links to both the formal publication through the relevant DOI and the license). See: https://creativecommons.org/licenses/by-nc-nd/4.0/.

\section{References}

1. Scanlon C, Mueller SG, Cheong I, et al. Grey and white matter abnormalities in temporal lobe epilepsy with and without mesial temporal sclerosis. J Neurol 2013;260:2320-9.

2. Bao Y, He R, Zeng Q, et al. Investigation of microstructural abnormalities in white and gray matter around hippocampus with diffusion tensor imaging (DTI) in temporal lobe epilepsy (TLE). Epilepsy Behav 2018;83:44-9.

3. Lemkaddem A, Daducci A, Kunz N, et al. Connectivity and tissue microstructural alterations in right and left temporal lobe epilepsy revealed by diffusion spectrum imaging. Neuroimage Clin 2014;5:349-58.

4. Pustina D, Avants B, Sperling M, et al. Predicting the laterality of temporal lobe epilepsy from PET, MRI, and DTI: A multimodal study. Neuroimage Clin 2015;9:20-31.

5. Shih YC, Tseng CE, Lin FH, et al. Hippocampal Atrophy Is Associated with Altered Hippocampus-Posterior Cingulate Cortex Connectivity in Mesial Temporal Lobe Epilepsy with Hippocampal Sclerosis. AJNR Am J 
Neuroradiol 2017;38:626-32.

6. Urbach H, Egger K, Rutkowski K, et al. Bilateral cingulum fiber reductions in temporal lobe epilepsy with unilateral hippocampal sclerosis. Eur J Radiol 2017;94:53-7.

7. Nazem-Zadeh MR, Elisevich K, Air EL, et al. DTIbased response-driven modeling of mTLE laterality. Neuroimage Clin 2015;11:694-706.

8. Fernández-Miranda JC, Rhoton AL Jr, Alvarez-Linera J, et al. Three-dimensional microsurgical and tractographic anatomy of the white matter of the human brain. Neurosurgery. 2008;62:989-1026; discussion 1026-8.

9. Wedeen VJ, Hagmann P, Tseng WY, et al. Mapping complex tissue architecture with diffusion spectrum magnetic resonance imaging. Magnetic Resonance in Medicine 2005;54:1377-86.

10. Johansen-Berg H, Rushworth MF. Using diffusion imaging to study human connectional anatomy. Annu Rev Neurosci 2009;32:75-94.

11. Schmahmann JD, Pandya DN, Wang R, et al. Association fibre pathways of the brain: parallel observations from diffusion spectrum imaging and autoradiography. Brain 2007;130:630-53.

12. Yeh FC, Wedeen VJ, Tseng WY. Estimation of fiber orientation and spin density distribution by diffusion deconvolution. Neuroimage 2011;55:1054-62.

13. Nigro S, Barbagallo G, Bianco MG, et al. Track density imaging: A reliable method to assess white matter changes in Progressive Supranuclear Palsy with predominant parkinsonism. Parkinsonism Relat Disord 2019;69:23-9.

14. Dai JK, Wang SX, Shan D, et al. Super-Resolution TrackDensity Imaging Reveals Fine Anatomical Features in Tree Shrew Primary Visual Cortex and Hippocampus. Neurosci Bull 2018;34:438-48.

15. Calamante F, Tournier JD, Jackson GD, et al. Trackdensity imaging (TDI): super-resolution white matter

Cite this article as: Wang $\mathrm{YH}$, Wang $\mathrm{ZM}$, Wei PH, Lu C, Fan XT, Ren LK, Shan YZ, Lu J, Zhao GG. Lateralizing the affected side of hippocampal sclerosis with quantitative high angular resolution diffusion scalars: a preliminary approach validated by diffusion spectrum imaging. Ann Transl Med 2021;9(4):297. doi: 10.21037/atm-20-5719 imaging using whole-brain track-density mapping. Neuroimage 2010;53:1233-43.

16. Fisher RS, Cross JH, D'Souza C, et al. Instruction manual for the ILAE 2017 operational classification of seizure types. Epilepsia 2017;58:531-42.

17. Papez JW. A proposed mechanism of emotion. 1937. J Neuropsychiatry Clin Neurosci 1995;7:103-12.

18. Bubb EJ, Metzler-Baddeley C, Aggleton JP. The cingulum bundle: Anatomy, function, and dysfunction. Neurosci Biobehav Rev 2018;92:104-27.

19. Garcia-Bengochea F, Corrigan R, Morgane P, et al. Studies on the function of the temporal lobes. I. The section of the fornix. Trans Am Neurol Assoc 1951;56:238-9.

20. Stylianou P, Hoffmann C, Blat I, et al. Neuroimaging for patient selection for medial temporal lobe epilepsy surgery: Part 1 Structural neuroimaging. J Clin Neurosci 2016;23:14-22.

21. Salmenpera TM, Simister RJ, Bartlett $P$, et al. Highresolution diffusion tensor imaging of the hippocampus in temporal lobe epilepsy. Epilepsy Res 2006;71:102-6.

22. Thivard L, Lehericy S, Krainik A, et al. Diffusion tensor imaging in medial temporal lobe epilepsy with hippocampal sclerosis. Neuroimage 2005;28:682-90.

23. Hakyemez B, Erdogan C, Yildiz H, et al. Apparent diffusion coefficient measurements in the hippocampus and amygdala of patients with temporal lobe seizures and in healthy volunteers. Epilepsy Behav 2005;6:250-6.

24. Fernandez-Miranda JC, Pathak S, Engh J, et al. Highdefinition fiber tractography of the human brain: neuroanatomical validation and neurosurgical applications. Neurosurgery 2012;71:430-53.

25. Maier-Hein KH, Neher PF, Houde JC, et al. The challenge of mapping the human connectome based on diffusion tractography. Nat Commun 2017;8:1349. 